\title{
$4 \mathrm{~V}$ \\ EXU Optical Design for a $100 \mathrm{~nm}$ CD Imaging System
}

\author{
D. W. Sweeney, R. Hudyma, \\ H. N. Chapman, and D. Shafer
}

This paper was prepared for and presented at the SPIE 23rd Annual International Symposium on Microlithography Santa Clara, California

February 22-27, 1998

April 9, 1998

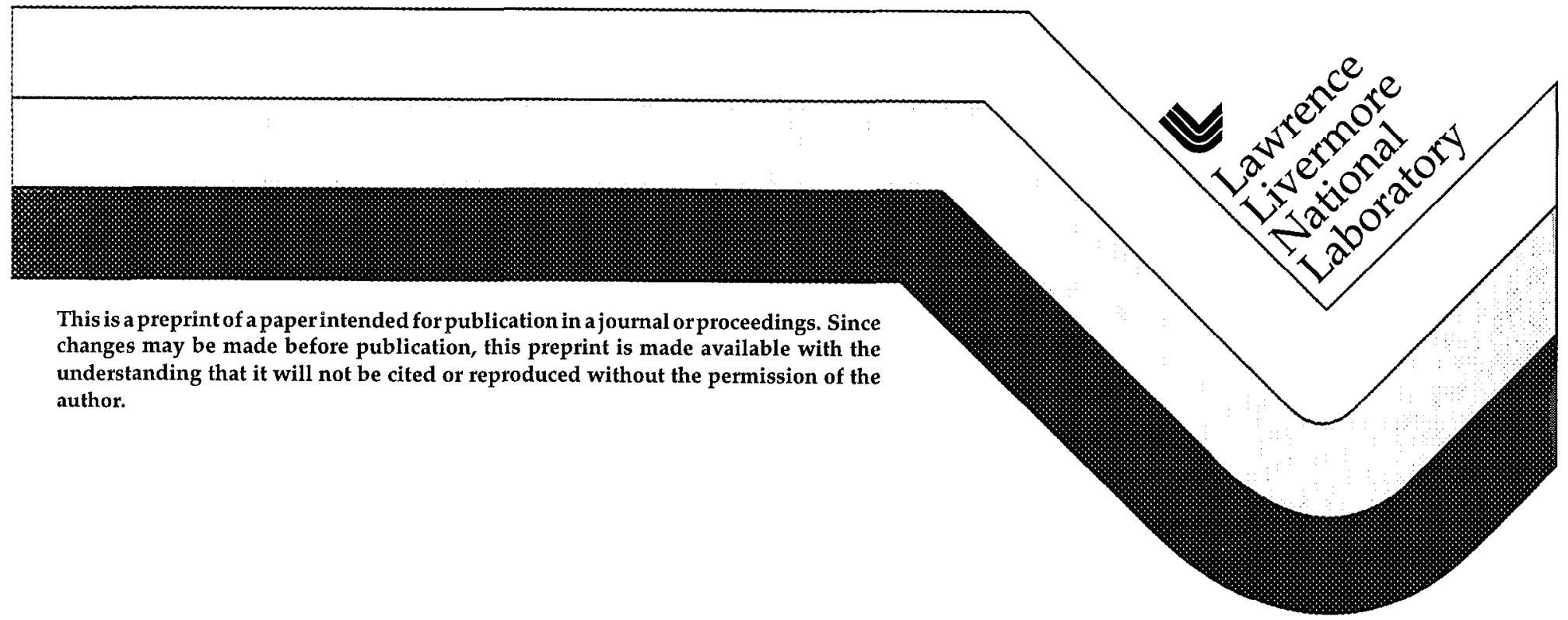




\section{DISCLAIMER}

This document was prepared as an account of work sponsored by an agency of the United States Government. Neither the United States Government nor the University of California nor any of their employees, makes any warranty, express or implied, or assumes any legal liability or responsibility for the accuracy, completeness, or usefulness of any information, apparatus, product, or process disclosed, or represents that its use would not infringe privately owned rights. Reference herein to any specific commercial product, process, or service by trade name, trademark, manufacturer, or otherwise, does not necessarily constitute or imply its endorsement, recommendation, or favoring by the United States Government or the University of California. The views and opinions of authors expressed herein do not necessarily state or reflect those of the United States Government or the University of California, and shall not be used for advertising or product endorsement purposes. 


\title{
EUV Optical Design for a $100 \mathrm{~nm}$ CD Imaging System
}

\author{
Donald W. Sweeney, Russ Hudyma, Henry N. Chapman, and David Shafer* \\ Lawrence Livermore National Laboratory, L-395, Livermore, CA 94550 \\ * David Shafer Optical Design, 56 Drake Lane, Fairfield, CT 06430
}

\begin{abstract}
The imaging specifications for extreme ultraviolet lithography (EUVL) projection optics parallel those of other optical lithographies. Specifications arc scaled to reflect the $100 \mathrm{~nm}$ critical dimension for the first generation EUVL systems. The design being fabricated for the Engineering Test Stand, an EUVL alpha tool, consists of a condenser with six channels to provide an effective partial coherence factor of 0.7 . The camera contains four mirrors; three of the mirrors are aspheres and the fourth is spherical. The design of the optical package has been constrained so that the angles of incidence and the variations in the angle of incidence of all rays allow for uniform multilayer coatings. The multilayers introduce a slight shift in image position and magnification. We have shown that a system aligned with visible light is also aligned at $13.4 \mathrm{~nm}$. Each mirror must be fabricated with an RMS figure error of less than $0.25 \mathrm{~nm}$ and better than $0.2 \mathrm{~nm}$ RMS roughness. Optical surfaces that exceed each of these specifications individually have been fabricated. The success of EUVL requires that these specifications be met simultaneously.
\end{abstract}

Keywords: EUV projection lithography, optical design, multilayer coatings, aspheric optics

\section{INTRODUCTION}

A consortium of semiconductor manufacturers has been formed to sponsor and participate in a program to develop extreme ultraviolet lithography (EUVL). The research is being conducted at a number of research facilities including substantial participation by Lawrence Livermore National Laboratory, Sandia National Laboratory, and Lawrence Berkeley National Laboratory. The collective goal of the three national laboratories is to develop and demonstrate the basic technologies required to produce an EUV lithographic-quality tool for $100 \mathrm{~nm}$ and smaller critical features. The laboratories are building an alpha-class tool that will operate at a wavelength of $13.4 \mathrm{~nm}$ with a 0.1 numerical aperture. This tool, called the Engineering Test Stand (ETS), is due for completion in late 1999 or early 2000. Figure 1 shows a representation of the condenser and camera for the ETS. This paper will present a description of the basic optics package for the ETS.

The basic precepts of EUV optical design will not be described here as excellent collections of papers on this topic are available in the literature ${ }^{1.23}$. The ETS camera design and its predicted performance is presented in detail in Section 2.0; the multilayer coating design is also included. Section 3.0 briefly describes alignment, although alignment is described more fully in a companion paper included in these proceedings ${ }^{4}$. Section 4.0 reviews the optical figure and finish specifications of the optical substrates.

\section{OPTICAL DESIGN}

The basic imaging specifications for the ETS camera are:

a. $100 \mathrm{~nm}$ critical dimension ( $70 \mathrm{~nm}$ control for isolated features) based on a numerical aperture N.A. of 0.1 , $\mathrm{K}_{1}=0.77$, and coherence factor $\sigma=0.7$.

b. Ring-field imaging with a cord-length of $26 \mathrm{~mm}$ at the wafer.

c. Fully stationary imaging with better than $1 \%$ intensity uniformity.

c. Depth of focus of $\pm 0.5 \mu \mathrm{m}$.

e. Reduction factor of $4: 1$ with residual magnification control of $\pm 20 \mathrm{ppm}$ and magnification resolution of 0.1 ppm.

f. Telecentric imaging at the wafer.

g. Total dynamic distortion of less that $5 \mathrm{~nm}$ over the full field. 


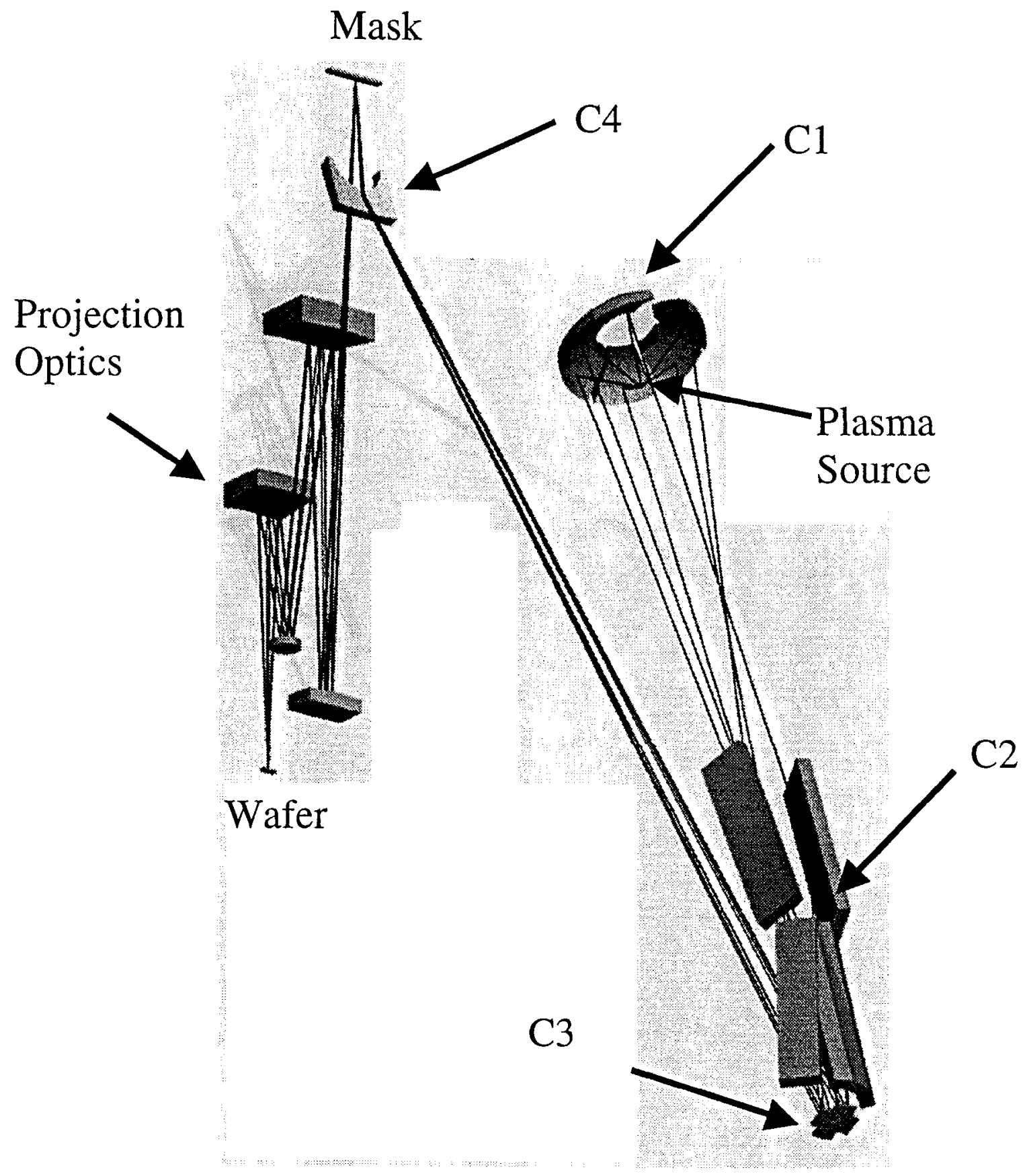

Figure 1. Basic optical layout of the ETS showing the plasma source, condenser, and projection optics. C1, C2, C3, and C4 are condenser elements. The size of the physical apertures in the camera are roughly to scale but the shape in actually more complex than shown.

The final ETS camera design with full parents is shown in Figure 2; this camera is currently under construction. The fabricated substrates will only include the clear apertures and edge regions. Design constraints require a minimum of $5 \mathrm{~mm}$ clearance between any clear aperture and neighboring rays. The basic design is a centered, ring-field system. The wellcorrected ring-field extends over a radial range from $208 \mathrm{~mm}$ to $214 \mathrm{~mm}$ in the mask plane. The overall length from the mask plane to the wafer plane is $1080 \mathrm{~mm}$.

The condenser system shown in Figure 1 consists of six parallel channels that in-total collect $30 \%$ of the radiation emitted by the laser produced plasma source. Each condenser channel can be divided into three functional groups: collector mirror $(\mathrm{C} 1)$, roof mirror pairs for field rotation (C2 and $\mathrm{C} 3$ ), and a field mirror (C4). The collector mirrors are 50 degree segments of a Compound Elliptical Concentrator; this element images a point to a segment of an arc. The roof-mirror pairs 
rotate the arcuate images produced by the CEC's so that they are aligned with the ring-field arc. The C2 mirrors are flats used in grazing incidence. The C3 mirrors are multilayer coated and used in near-normal incidence. The C3 mirrors are located at the condenser pupil. The field mirror (which is the common last element of all six channels) is a toroid that images the condenser pupil onto the projection optics entrance pupil. The condenser package provides an effective coherence factor of 0.7 .

The camera consists of four mirrors labeled M1, M2, M3, and M4 in Figure 2. Three of the four mirrors are aspheric, M3 is a sphere. The aperture stop is essentially located at Mirror 3. M1 and M3 are convex; M2 and M4 are concave. Each mirror surface is characterized by its base radius and, as necessary, by aspheric coefficients according to the equation,

$$
z(r)=\frac{c r^{2}}{1+\sqrt{1-(1+k) c^{2} r^{2}}}+A r^{4}+B r^{6}+C r^{8}
$$

where $z(r)$ is the surface sag parallel to the $z$-axis as a function of the radial distance $r$ from the optical axis; $c$ is the surface curvature and equals the reciprocal of the base radius; $k$ is the conic constant; and $\mathrm{A}, \mathrm{B}$, and $\mathrm{C}$ are polynomial coefficients.

Each mirror in the camera must be multilayer coated to reflect $13.4 \mathrm{~nm}$ radiation. The multilayers consist of 40 alternating layers of molibdinum and silicon. The reflectivity of the Mo-Si multilayer is typically $67 \%$. Care has been taken in the camera design to allow uniform multilayers to be coated onto the surface of each optic. Mirrors M1, M2, and M4 have

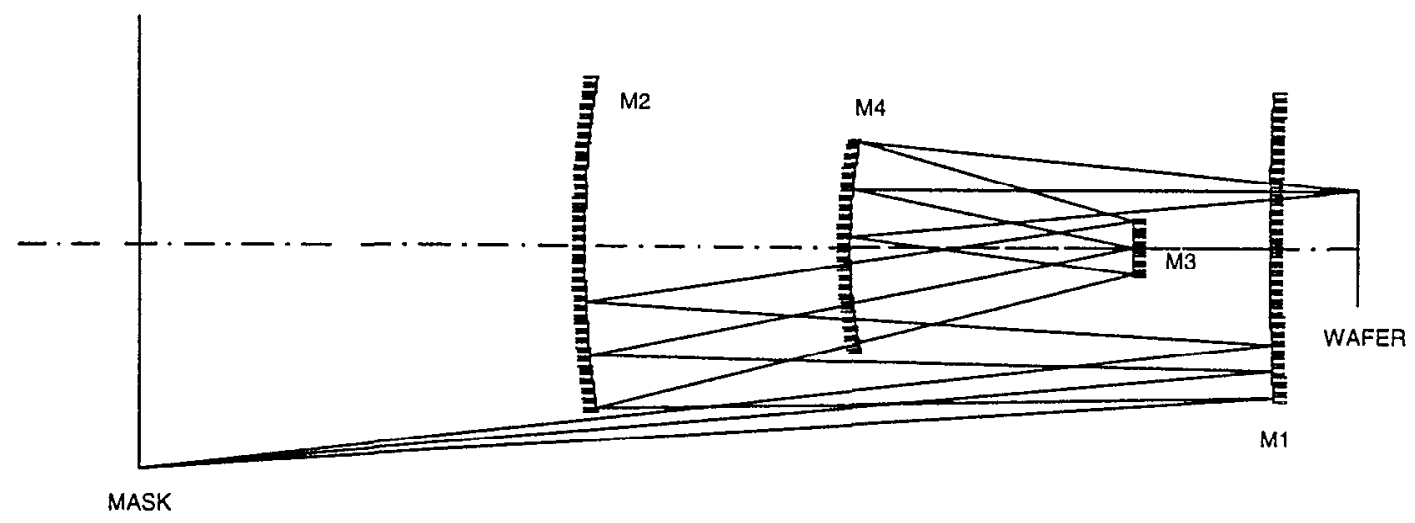

Figure 2. Projection optics layout for ETS design showing the full parent optics.

identical multilayer coatings of,

$$
\mathrm{Mo}(2.92 \mathrm{~nm}) / \mathrm{Si}(4.0 \mathrm{~nm}) \times 40 .
$$

Mirror M3 has a higher average angle of incidence and has a slightly different coating,

$$
\operatorname{Mo}(2.92 \mathrm{~nm}) / \mathrm{Si}(4.12 \mathrm{~nm}) \times 40 .
$$

Calculations show that the nominal multilayer coatings do not degrade the quality of the exit pupil wavefront.

\subsection{Aberration correction and performance summary}

During the camera design optimization, the primary constraints located the diffraction centroid and controlled the telecentricity of the imaging bundles at the wafer. The aspheric sag of the mirrors was constrained to be less than $10 \mu \mathrm{m}$ as measured over the used clear aperture of the parent. Angles of incidence (AOI) were constrained on each mirror to ensure compatibility with the desired uniform multilayer. Both the mean angle of incidence and the range of angles on each mirror were controlled. Packaging constraints to control ray bundle clearance and working distances were also used. 


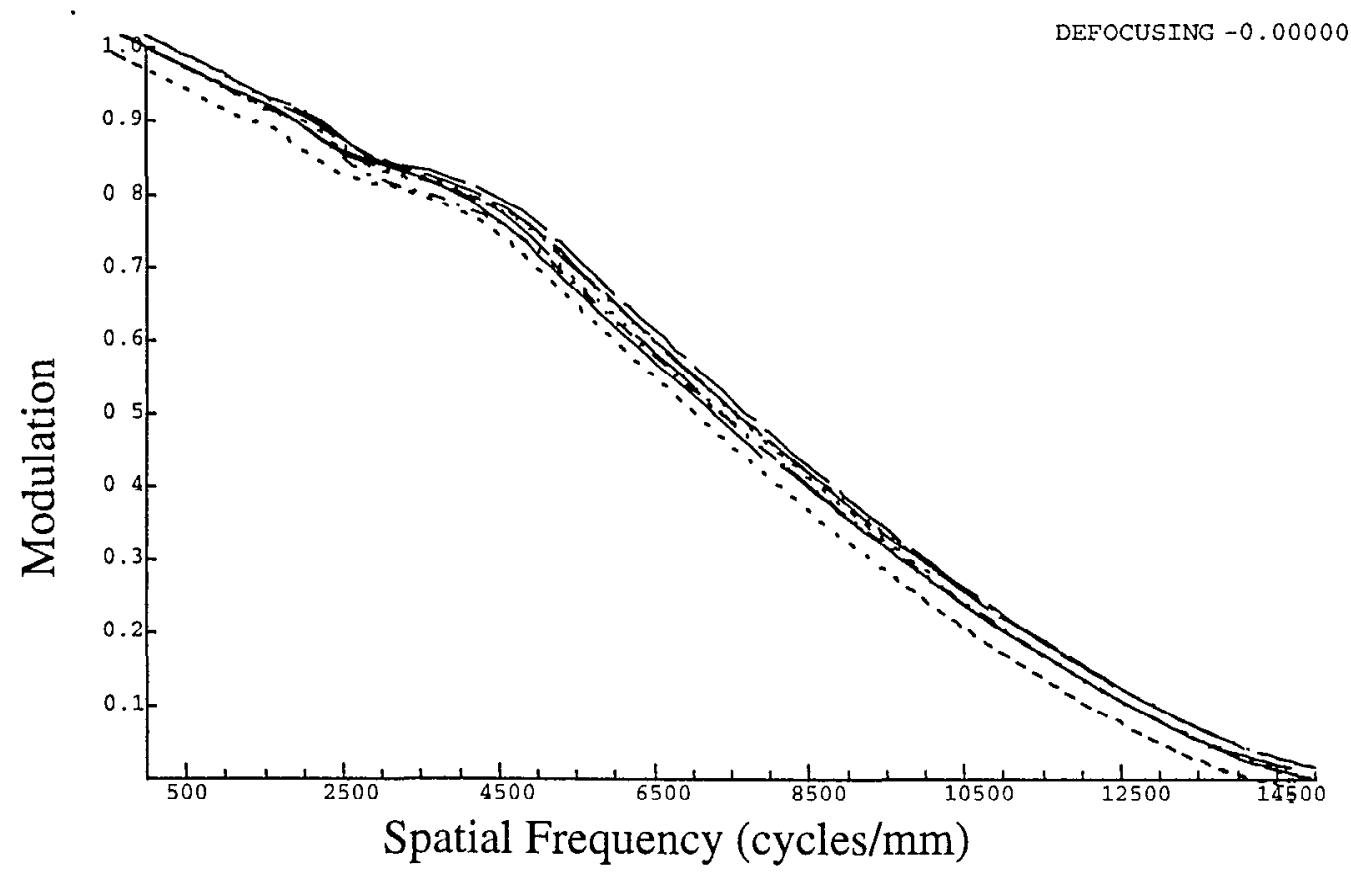

Figure 3. Incoherent square wave response for the ETS design.

A four component system has sufficient degrees of freedom to control the reduction ratio, wavefront error, telecentricity, field curvature, and distortion. A four mirror system can benefit from a negative primary followed by a positive focusing group much in the same way its refractive counterpart, the inverted telephoto, benefits from this same firstorder construction. The negative front group (in this embodiment the primary mirror M1) reduces the field angles presented to the positive focusing group. This reduction in ray angles will naturally lead to lower higher-order aberration residuals.

Since this is a highly corrected optical system, the image quality is characterized by the magnitude of the wavefront deformations rather than on the nature of the individual aberrations. To reduce the design residuals, a merit function that minimizes the variance of the wavefront error is used. When minimizing the wavefront variance, aberrations that do not blur the image but only shift its location (distortion) become harder to control. At the level of EUVL performance, it is quite difficult to develop constraints that will influence the centroid location (i.e., distortion), while simultaneously allowing for improved RMS wavefront error performance. The design achieves near diffraction-limited performance at a 0.1 numerical aperture. The MTF is shown in Figure 3 for the center and both edges of the ring field out to $15000 \mathrm{lp} / \mathrm{mm}$.

Astigmatism drives the aberration correction of the system. The ring-field is centered about the location where the astigmatism is zero. The astigmatism node is clearly depicted in Figure 4. The field curves show the $5^{\text {th }}$ and $7^{\text {th }}$ order astigmatism balance that is achieved by adding the $6^{\text {th }}$ and $8^{\text {th }}$ order deformations to M1, M2, and M4. In a global sense, it is the state of this $5^{\text {th }}$ and $7^{\text {th }}$ order balance that actually places an upper limit on the width of the ring that can be achieved with designs of this form.

The distortion field plot (see Figure 4) shows a balance between $3^{\text {rd }}$ and $5^{\text {th }}$ order distortion; the $7^{\text {th }}$ order distortion plays less of a role. Tailoring the balance between the $3 \mathrm{rd}$ and 5 th order contributions minimizes static distortion. Locating the point of inflection of the distortion curve at the astigmatism node minimizes the rate of change of magnification within the ring field and naturally reduces the peak-to-valley distortion to a minimum. Conventional thought might reason that the astigmatism and distortion nodes should be designed to coincide in the ring field. Given the fact that higher order aberrations will dominate the residual errors in a field-biased system, this is not the case. This is easily observed in Figure 4 . At the distortion node, the change in magnification across the ring would be unacceptable, even though the distortion is identically zero at one point as shown in the plot. Note that we will have the ability to calibrate the distortion, which is equivalent to shifting the origin of the distortion plot anywhere we wish along the distortion curve. 
Wide-angle all reflective systems arc typically limited by either oblique spherical aberration or elliptical coma. These higher-order residuals may be seen from the Zernike wavefront decomposition shown in Table 1. The elliptical coma (Z10/Z11) limits the performance of the design on both sides of the ring field. The design has contributions which are nearly equal and opposite. Forcing the elliptical coma to zero on one side of the field will tend to increase the elliptical coma on the other. Allowing third-order coma (Z7/Z8) of opposite sign mitigates this fifth-order coma. This small third-order overcorrected residual does not arise from the comatic balance that is struck between $\mathrm{M} 1 / \mathrm{M} 3$ and $\mathrm{M} 2$; it is interesting to note

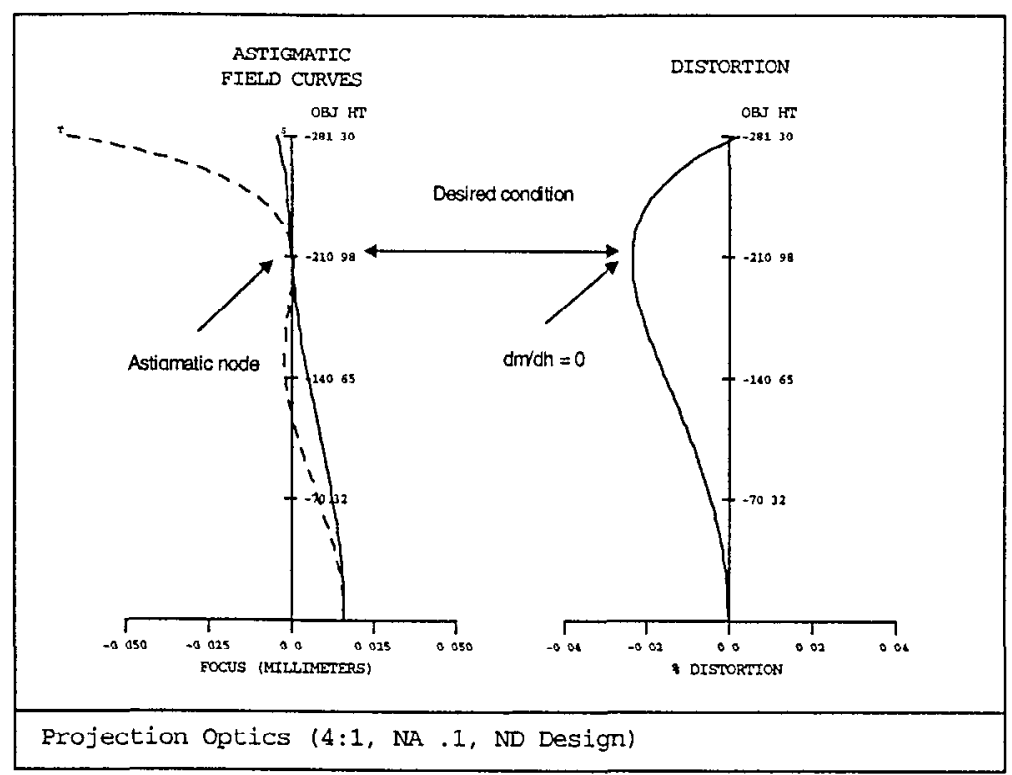

Figure 4. Field curves illustrating nature of astigmatism and distortion correction. Also see Figure 6 that shows the static distortion in nanometers across the ring field.

that the conic on M4 leaves this third-order residual. The primary elliptical coma contribution comes from M4.

\subsection{Distortion}

Patterns will be printed with the projection optics by continuously scanning the mask and wafer in synchrony. This has a substantial effect on the image aberrations, particularly distortion. In general, the distortion of the scanned image is much reduced as compared with the static image. However, a variation of the static distortion along the path of a scanned point leads to blurring, which degrades the quality of the printed image. The printed image properties will be field-invariant in the scan direction, but will vary in the perpendicular direction. This variation is due to the fact that the static distortion field of the design displays radial symmetry, i.e., the distortion vector either points away from or toward the optic axis. The linear scan of the mask and wafer breaks this symmetry. The breaking of the symmetry becomes more apparent when the ratio of the wafer and mask velocities do not match the reduction ratio of the projection optics. If, for example, the mask is scanned too fast in a direction from the inside of the ring to the outside, then a line on the mask which is perpendicular to the scan direction would be printed curved, with the ends of the line printed ahead of its center.

The effect of the static distortion field on the printed image can be examined by computing the trajectory that the image of a mask point makes on the wafer. Trajectories of the centroid of images of field points on the mask are shown in Figure 5. The plotting symbols mark out intervals of equal time. Due to the fact that the static distortion field varies quadratically with radial distance from the optic axis, the image moves with a constant acceleration relative to the moving wafer. The 
projection optics have been designed so that the point of inflection of the radial static distortion field is in the center of the ring field, which causes the image to slow down to zero velocity half-way along its trajectory. The printed image, which is the time average of the moving image, is therefore heavily weighted to a state of low distortion. It is found that in this case of "balanced" static distortion, the peak-to-valley static distortion of $16 \mathrm{~nm}$ (see Figure 6) leads to a scan blur which gives a Strehl ratio of 0.993 for the printed image.

The centroids of the scan trajectories give the locations of the printed image points. The variation of these locations is referred to as dynamic distortion. As shown in Figure 5(a), the dynamic distortion causes a horizontal line to be primarily stretched in the horizontal direction. At the edge of the field, the dynamic distortion is $1 \mathrm{~nm}$ in the cross-scan direction and only $0.13 \mathrm{~nm}$ in the scan direction. The dynamic distortion in the cross-scan direction can be corrected by trimming the magnification of the projection optics by $0.08 \mathrm{ppm}$, which will increase the dynamic distortion in the scan direction to 0.35 $\mathrm{nm}$. The effect of an error in scan velocity of the wafer by $1.0 \mathrm{ppm}$ is shown in Figure $5(\mathrm{~b})$.

\subsection{Telecentricity}

The design is telecentric at the wafer but not at the reflective mask. The fidelity of the telecentric condition was determined by analyzing the variation in centroid location through-focus for several points in the ring field. As the focus is shifted longitudinally by $2 \mu \mathrm{m}( \pm 1 \mu \mathrm{m})$, the centroid shifts $0.02 \mathrm{~nm}$ at the bottom and $-0.16 \mathrm{~nm}$ at the top of the ring, respectively. This equates to a telecentricity error that causes a change in image height of $0.01 \mathrm{~nm} / \mu \mathrm{m}$ and $-0.08 \mathrm{~nm} / \mu \mathrm{m}$ at the bottom and top of the ring, respectively.

\subsection{Variation in angles of incidence at the mirrors}

To ensure compatibility with the current multilayer designs, the mean angle of incidence (AOI) and the variation in AOI were constrained during the detailed optimization effort. When considering coating performance, the angles from all the points in the field must be examined. Analysis shows that M3 supports the largest angle of incidence at 12.9 degrees. The range in angles on $\mathrm{M} 3$ is approximately 1.9 degrees.

\section{CAMERA ALIGNMENT}

The camera will be assembled using interferograms and distortion measurements made at a number of field points [see Reference 4]. The multiconjugate aberration data describe the state of the optical system, and the corrections that are required are inferred from these. The alignment procedure will be an iterative Newton-type method, whereby the corrections are determined from a linear model relating aberrations to the rigid-body degrees of freedom of the optical elements. The linear model is in fact the sensitivity matrix of the optical system. At each iteration step, the aberration field is remeasured and the corrections of compensators are made. The inverse problem of finding the corrections, given the aberrations, is illconditioned and requires regularization. This is achieved using the singular value decomposition (SVD) of the sensitivity matrix of the optics.
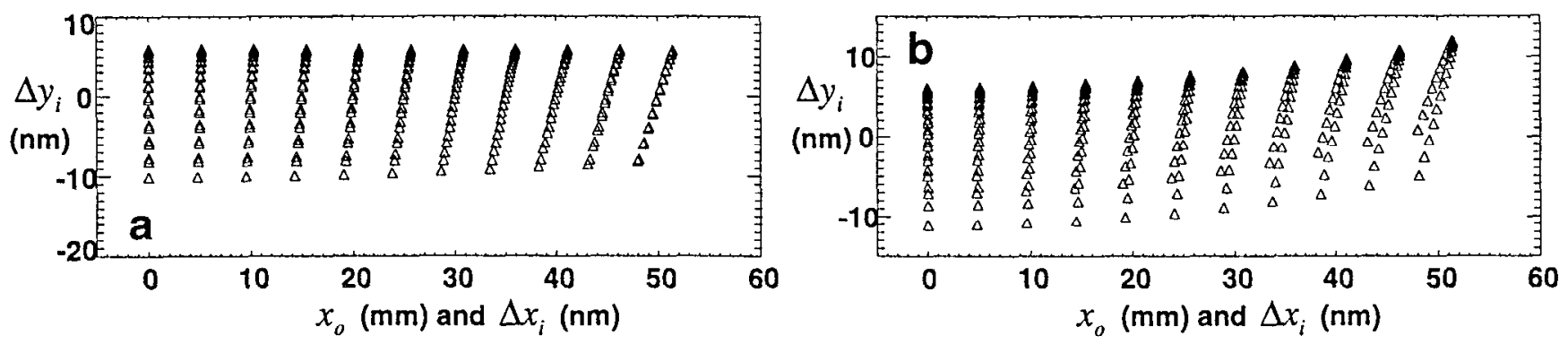

Figure 5. Trajectories of the centroid of images of field points as they move through the ring-field aperture, (a) when the wafer is scanned 0.25 the speed of the mask and (b) when the wafer is scanned $1 \mathrm{ppm}$ slower. The image trajectory is $\left(\Delta x_{i}, \Delta y_{i}\right)$, and varies as a function of the object field position, $x_{0}$, relative to the center of the ring-field. 


\begin{tabular}{|c|c|c|c|c|}
\hline & \multicolumn{2}{|c|}{ Polynomial Coefficients } & $\begin{array}{c}\text { Rorresponding } \\
\text { Aberration }\end{array}$ \\
\hline Term & $\begin{array}{c}\text { Ring } \\
\text { Bottom }\end{array}$ & $\begin{array}{c}\text { Ring } \\
\text { Center }\end{array}$ & $\begin{array}{c}\text { Ring } \\
\text { Top }\end{array}$ & Piston \\
\hline 1 & -0.009 & -0.006 & 0.004 & Tip \\
\hline 2 & 0.000 & 0.000 & 0.000 & Tilt \\
\hline 3 & -0.104 & -0.018 & 0.064 & Defocus \\
\hline 4 & 0.002 & -0.003 & -0.001 & Astigmatism \\
\hline 5 & -0.002 & 0.007 & -0.001 & Astigmatism \\
\hline 6 & 0.000 & 0.000 & 0.000 & Third-order Coma \\
\hline 7 & 0.000 & 0.000 & 0.000 & Third-order Coma \\
\hline 8 & -0.043 & 0.000 & 0.040 & Spherical \\
\hline 9 & 0.009 & 0.000 & -0.008 & Elliptical coma \\
\hline 10 & 0.000 & 0.000 & 0.000 & Elliptical coma \\
\hline 11 & -0.033 & 0.001 & 0.039 & Oblique spherical aberration \\
\hline 12 & 0.001 & 0.002 & 0.003 & Fifth-order coma \\
\hline 13 & 0.000 & 0.000 & 0.000 & Oblique spherical aberration \\
\hline 14 & 0.000 & 0.000 & 0.000 & Fifth-order coma \\
\hline 15 & 0.005 & 0.005 & 0.004 & RMS of polynomial \\
\hline & 0.056 & 0.010 & 0.038 & RMS w/tilt removed \\
\cline { 2 - 5 } & 0.020 & 0.004 & 0.020 & \\
\cline { 2 - 5 }
\end{tabular}

Table 1. Fringe Zernike decomposition of wavefront error through fifth-order. Units are waves at $13.4 \mathrm{~nm}$

The alignment will be achieved by adjusting only a set of predetermined compensators. The compensators are the smallest number of rigid-body degrees of freedom that can address all the possible misalignment aberrations in the most independent way. An adjustment is chosen as a compensator not on the basis of what aberration it alone can correct, but on how it complements the other compensators. Selecting the compensators is not straightforward since the interrelationships between adjustments is complex, a consequence of the aspheric optical surfaces. The method of compensator selection is described in Reference 4. It is important to keep the number of compensators required for alignment to a minimum, so the structural support of the optics can be kept as stiff and stable as possible. It is found that the minimum number of compensators increases logarithmically with the magnitude of the aberration that must be corrected. In particular, each time an additional optimally-chosen compensator is included the correctable aberration increases by a factor of 1.8 .

The aberration measurements must be made to an accuracy that is comparable or better than the residual wavefront error that can be tolerated in the assembled system. It is found that to align the system to $\lambda / 20 \mathrm{RMS}$ for EUV operation requires aberration measurements accurate to $0.27 \mathrm{~nm}$ RMS. This can be achieved with the visible light using the so-called Sommargren interferometer ${ }^{5}$. Even though the visible testing wavclength is vastly different from the EUV operating wavelength, analysis has shown that the projection optical system is truly achromatic. The EUV light reflects from the optics by a resonance from all layers in the multilayer coating, whereas visible light only interacts with the top layers. In principle, the phase change on reflection may be considerably different for EUV than visible. However, in this optical design, the phase difference on reflection from any one mirror is small, due to the well-constrained angles of incidence, and the visiblelight phase errors from each mirror tend to compensate each other. The only difference in wavefront between the two wavelengths is a small tilt, causing a magnification change as shown in Figure 6. Thus, a projection optical system aligned using visible light will also be aligned at EUV.

\section{OPTICAL FABRICATION}

The specifications for the ETS mirror substrate figure and finish are shown in Table 2. Optical surfaces that approach these specifications have been fabricated. The success of EUVL requires that these figure and finish specifications be simultaneously met. 


\begin{tabular}{|c|c|c|}
\hline Component & Specification & Definition \\
\hline Surface figure & $<0.25 \mathrm{~nm}$ RMS & $\begin{array}{c}\text { Integration of PSD for } \\
\text { frequencies between (clear } \\
\text { aperture })^{-1} \text { and } 1 \mathrm{~mm}^{-1}\end{array}$ \\
\hline $\begin{array}{c}\text { Mid-spatial } \\
\text { frequency } \\
\text { roughness }\end{array}$ & $<0.20 \mathrm{~nm}$ RMS & $\begin{array}{c}\text { Integration of PSD for } \\
\text { frequencies between } 1 \mathrm{~mm} \\
\text { and } 1 \mu \mathrm{m}^{-1}\end{array}$ \\
\hline $\begin{array}{c}\text { High-spatial } \\
\text { frequency } \\
\text { roughness }\end{array}$ & $<0.10 \mathrm{~nm}$ RMS & $\begin{array}{c}\text { Integration of PSD for } \\
\text { frequencies }>1 \mu \mathrm{m}^{-1}\end{array}$ \\
\hline
\end{tabular}

Table 2. Specifications for aspheric mirror substrates.

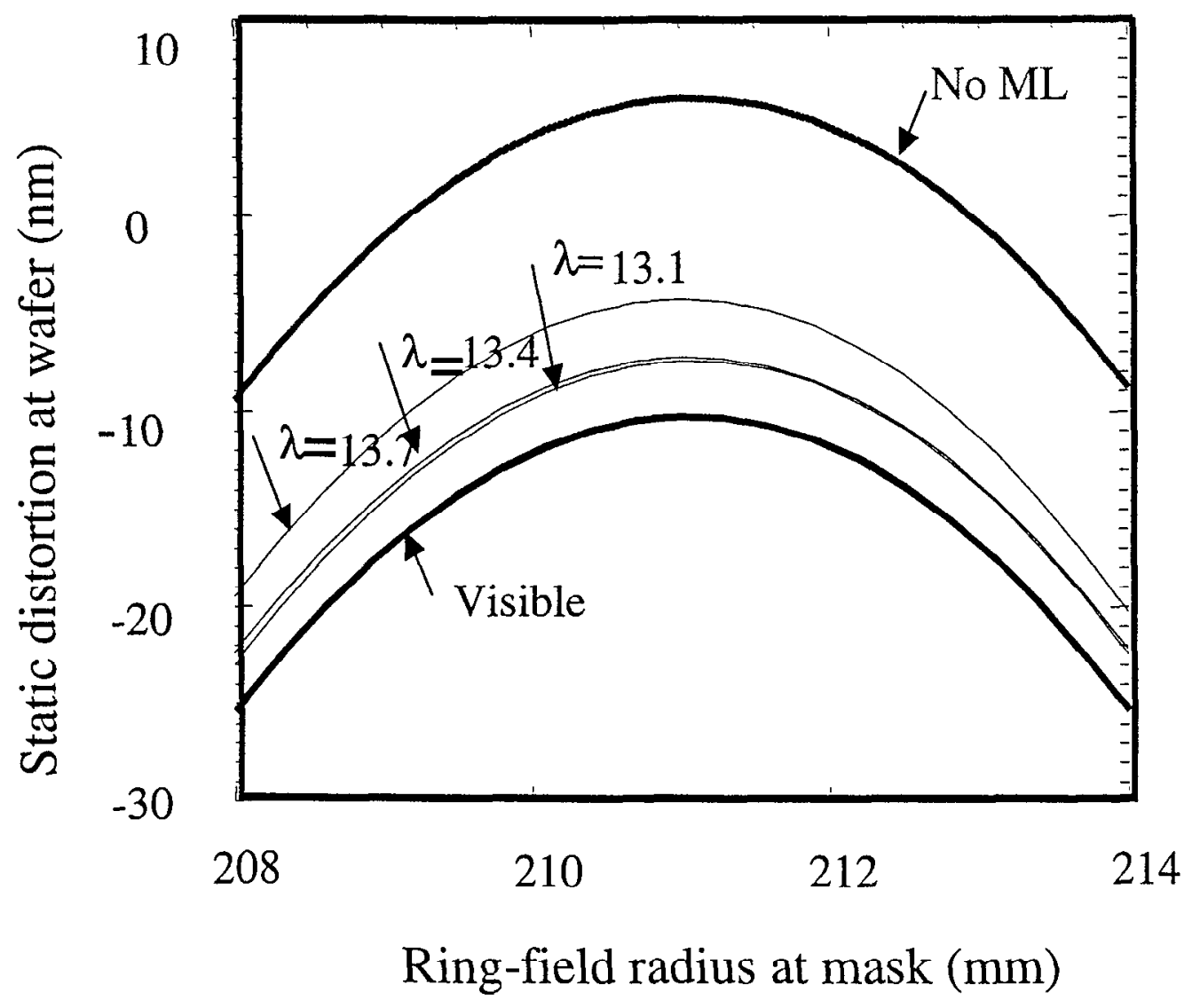

Figure 6. Static distortion across the ring field. Rays are traced through the system when no multilayer is present, when the EUV multilayer is present, and when visible light is used. 
The figure specifications in Table 2 are measured relative to the optical fabricator's metrology. Any errors in the vendor's metrology are embedded in the fabricated part. The current EUVL optical fabrication process delivers Sommargren interferometers ${ }^{5}$ directly to the optical supplier's fabrication facilities. These interferometers are capable of an absolute accuracy for single aspheres of $0.1 \mathrm{~nm}$ RMS.

The mirror substrates must maintain their figure when they are coated, installed, and operated in the camera. Issues such as residual stress introduced during coating, thermal loading during imaging, and residual mounting forces have all been addressed. Reliable procedures exist for handling these factors.

\section{CONCLUSIONS}

This paper has presented the basic optical design of the Engineering Test Stand. Using a wavelength of $13.4 \mathrm{~nm}$ and a numerical aperture of 0.1 , the system will easily support a lithographic critical dimension of $100 \mathrm{~nm}$. The basic design configuration is a scanning ring-field. The ring-field is $6 \mathrm{~mm}$ wide by $104 \mathrm{~mm}$ in the mask plane and is reduced by a factor of four in the wafer plane. The camera has four, multilayer coated mirrors; three of these mirrors are aspheric. The condenser has six independent channels that together produce an effective partial coherence fill factor $\sigma=0.7$.

Of course, fabrication of the optical substrates, multilayer coating of the substrates, optical alignment, and stage scanning are just a few of the practical technical challenges that must be resolved to ficld a working EUV lithographic tool. This paper has commented on a few of these issues. A number of the following papers in this proceedings deal with meeting these challenges.

\section{ACKNOWLEDGEMENTS}

This work was performed under the auspices of the U.S. Department of Energy by the Lawrence Livermore National Laboratory under Contract No. W-7405-ENG-48. Funding was provided by the Extreme Ultraviolet Limited Liability Corporation under a Cooperative Research and Development Agreement.

\section{REFERENCES}

1. OSA Proceedings on Soft-X-Ray Projection Lithography, Vol. 12, J. Bokor, ed. (Optical Society of America, April 1012, 1991, Monterey, California).

2. OSA Proceedings on Extreme Ultraviolet Lithography, Vol. 23, F. Zernike and D. T. Atwood, eds. (Optical Society of America, September 19-21, 1994, Monterey, California).

3. OSA Trends in Optics and Photonics Vol. 4, Extreme Ultraviolet Lithography, G. D. Kubiak and D. R. Kania, eds. (Optical Society of America, Washington, DC 1996)

4. H. N. Chapman and D. W. Sweeney, "A Rigorous Method for Compensation Selection and Alignment of Microlithographic Optical Systems," in these proceedings.

5. G. E. Sommargren, "Diffraction methods raise interferometer accuracy," Laser Focus World 32, pp. 61-71, August 1996. 


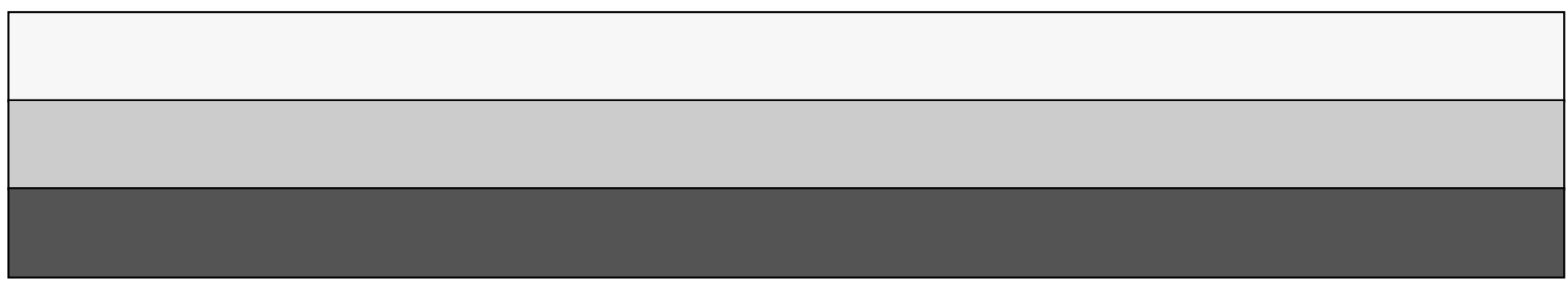

